\title{
A Wikipédia como diálogo entre universidade e sociedade: uma experiência em extensão universitária
}

\author{
Juliana Bastos Marques ${ }^{1}$, Otavio Saraiva Louvem² \\ ${ }^{1}$ Departamento de História, Centro de Ciências Humanas e Sociais - Universidade \\ Federal do Estado do Rio de Janeiro (UNIRIO) \\ Av. Pasteur, 458 - 22290-240 - Rio de Janeiro, RJ - Brasil \\ ${ }^{2}$ Petrobrás - Petróleo Brasileiro S. A. \\ Av. República do Chile, 65 - 20031-912 - Rio de Janeiro, RJ - Brasil \\ juliana.marques@unirio.br, otavio1981.wiki@gmail.com
}

\begin{abstract}
Resumo. $O$ artigo apresenta uma experiência no trabalho com o uso crítico e edição de artigos da Wikipédia lusófona no ambiente universitário, em atividades de extensão, realizado na Universidade Federal do Estado do Rio de Janeiro (Unirio) em 2012. Foram realizados diferentes tipos de atividades, desde workshops de 4 h até cursos de maior duração, tanto para o público adulto geral quanto para universitários segmentados por área de estudo. $O$ objetivo do trabalho foi exercitar competências críticas de leitura e produção de textos de divulgação, trazendo e adaptando para o usuário da Wikipédia conhecimentos ensinados em nível de graduação e pós-graduação.
\end{abstract}

\begin{abstract}
The paper presents an experience with critical reading and edition of Portuguese Wikipedia articles in the university, in extension activities, conducted at the Federal University of Rio de Janeiro State (Unirio), in 2012. Different types of activities were introduced, from $4 h$ workshops to longer term courses, for both broader audiences and university students by field of study. The goal of the activities was to excercise critical proficiency in reading and writing skills, offering and adapting for the regular Wikipedia user academic knowledge produced in undergraduate and graduate levels.
\end{abstract}

\section{Introdução}

A Wikipédia tem sido utilizada já há mais de uma década, e ainda com crescente frequência, como fonte de informação e conhecimento para estudantes de todos os níveis de ensino, desde o Fundamental até a pós-graduação. Embora seu uso seja geralmente desencorajado pelos professores, que veem o site como uma fonte não muito confiável [Vieira 2008], a combinação entre a facilidade de acesso aos artigos - é geralmente o primeiro resultado para definições de termos em buscas no Google - e a substituição cada vez maior do hábito de leitura e busca de informações em livros impressos para mídias digitais [Dudziak 2008] fazem com que a Wikipédia tome um espaço sem precedentes como material didático e informativo. Somado a isso, a enciclopédia online tem sido cada vez mais utilizada como referência primordial entre usuários fora do mundo escolar e acadêmico, de jornalistas a curiosos em geral. De fato, 
apenas na Wikipédia em português (http://pt.wikipedia.org) tem-se uma média mensal de 478 milhões de visitas por página, o que equivale a quase 16 milhões de visitas por dia $^{1}$.

A ubiquidade sem perspectiva próxima de declínio de seu uso torna, assim, imperativo que se faça desse recurso uma fonte de qualidade, embora, devido à sua própria natureza, a Wikipédia não disponha de um dispositivo editorial que garanta isso [Ayers et al. 2008; Santos \& Pandini 2009]. Como condição definidora de seu funcionamento, a plataforma wiki permite que qualquer pessoa possa editar os artigos da enciclopédia. No entanto, diferentemente de uma percepção generalizada entre seus usuários, mesmo qualificados [Soylu 2009], a Wikipédia não é um território de ninguém, pois há diversos mecanismos de controle e correção, definidos por consenso entre seus editores e com dinâmicas específicas para as comunidades de cada língua na qual a Wikipédia é escrita [Rosado 2007; Serrano 2009]. As cinco regras comuns a todas essas comunidades, conhecidas como "pilares", são guias normativos que regem a dinâmica dessa produção colaborativa e anônima, a partir das quais se pretende paulatinamente - dado o tamanho e a velocidade das edições - providenciar confiabilidade às informações apresentadas no site. Esses pilares são'

1. "A Wikipédia é uma enciclopédia": o que se pressupõe a definição de enciclopédia como o conjunto de textos introdutórios a assuntos de relevância de alguma forma determinável, e que compõem um amálgama de conhecimentos previamente conhecidos e publicados sobre os assuntos sobre os quais versa;

2. "A Wikipédia rege-se pela imparcialidade": um corolário da definição moderna de enciclopédia [Salsano 2000], em que todos os pontos de vista sobre determinado assunto são apresentados de maneira equilibrada, sem julgamentos de valor e devidamente referenciados por fontes externas verificáveis, pois estas sim são autorais;

3. “A Wikipédia é uma enciclopédia de conteúdo livre": editável por qualquer pessoa e de disponibilidade gratuita, ela pode ser replicada em outros textos, desde que propriamente citada como fonte ${ }^{3}$;

4. "A Wikipédia possui normas de conduta", ponto que a reconhece como próxima às redes sociais, no sentido em que estabelece uma dinâmica própria de interação social entre editores a princípio anônimos, e que deve funcionar com cordialidade [Campos 2009; D'Andréa, 2012];

5. "A Wikipédia não possui regras fixas", pilar também conhecido como "seja audaz". De fato, a entidade mantenedora do site, a Wikimedia Foundation, não se responsabiliza pela forma como cada comunidade em cada língua se organiza e gere os artigos editados. Cabe aos próprios editores criar e administrar as

http://stats.wikimedia.org/EN/SummaryPT.htm. Mais informações globais em

${ }^{2}$ http://pt.wikipedia.org/wiki/Wikipédia:Cinco_pilares. Acessado em 30 de setembro de 2013.

${ }^{3}$ As licenças usadas pela Wikipédia são GFDL (GNU Free Documentation License) e CC-BY-SA 3.0 (http://creativecommons.org/licenses/by-sa/3.0/br/, acessado em 30 de setembro de 2013.). 
enciclopédias de cada língua [Casebourne et al. 2012] de maneira a chegar em consenso.

Para fomentar a expansão de um trabalho de qualidade e aumentar o número de editores, a Wikimedia Foundation passou, em 2010, a trabalhar em conjunto com algumas universidades, a princípio nos EUA e em seguida em outros países [Obar \& Roth 2011; Roth et al. 2013]. Algumas iniciativas acadêmicas individuais já haviam sido iniciadas [Cummings 2009], mas o apoio da fundação permitiu uma integração maior entre os três polos fundamentais no trabalho de edição da Wikipédia dentro da universidade, ou seja, professores, alunos e editores experientes - já que estes já possuíam um conhecimento mais aprofundado das regras, dos códigos wiki e da dinâmica das comunidades que administram cada versão. No programa idealizado pela fundação, editores voluntários poderiam trabalhar em conjunto com professores (o "Programa Embaixadores" $)$, como monitores presenciais ou à distância, auxiliando os alunos nos meandros técnicos do site. Para tudo isso, as metodologias de trabalho ainda são incipientes e empíricas, já que a iniciativa seria uma experiência inédita de trabalho conjunto entre estudantes universitários e uma gama diversa de internautas anônimos, das mais diversos proveniências e níveis de conhecimento [Watts 2012].

Mas para quê usar a Wikipédia como ferramenta de construção de textos em sala de aula? As iniciativas que se acumularam demonstraram diversos pontos positivos para a formação de competências e habilidades em alunos das mais diversas áreas de conhecimento. Em primeiro lugar, reverte-se a postura passiva inicial do estudante em usar a Wikipédia como fonte indiscriminada e acrítica de informação: o primeiro passo, ler e decodificar criticamente o texto de um determinado artigo, seguindo os pressupostos e consequências dos dois primeiros pilares, faz com que o aluno desenvolva uma capacidade de discernimento mais apurada sobre a natureza da informação, sua confiabilidade e verificabilidade [Saorín et al. 2011; Lampe et al. 2012]. Sendo ele mesmo o produtor do texto, o aluno se torna responsável por essa mesma confiabilidade, e também passa a compreender o problema do plágio e da cópia indiscriminada de informações [Souza 2007; Silva \& Domingues 2008] - questão relacionada ao terceiro pilar. Ao praticar a escrita colaborativa, o aluno interage de maneira mais aprofundada com seus pares, já que de várias formas todos participam da leitura, produção e avaliação dos artigos produzidos, mas também aprendem a dialogar com editores anônimos externos ao seu ambiente, absorvendo a percepção de que não são donos do conhecimento produzido e podem perfeitamente aceitar uma contribuição externa - ou deverão saber argumentar com propriedade caso queiram corrigir uma edição feita por outrem que julguem errônea (quarto pilar) [Serrano 2009].

Por fim, diferentemente de iniciativas pedagógicas com wikis fechadas [Gomes 2007; Barbosa \& Oeiras 2008; Bottentuit \& Coutinho 2008; Jesus 2011; Costa et al. 2011], o resultado final do trabalho em aula é revertido de maneira pública, o que alguns estudos e verificações empíricas recentes têm mostrado que aparece como elemento motivador de significativa relevância para os estudantes [Lampe et al. 2012; Watts 2012; cf. Costa \& Passerino 2011]. Em última instância, os alunos estão produzindo, em diferentes graus de complexidade, o próprio material didático que eles e seus colegas utilizam.

\footnotetext{
${ }^{4}$ http://outreach.wikimedia.org/wiki/Wikipedia_Ambassador_Program. Acessado em 30 de setembro de 2013.
} 
Assim, o trabalho com a Wikipédia no ambiente universitário se mostra um canal de comunicação bastante eficiente entre o conhecimento que é aprendido e produzido dentro da universidade e as demandas de informação da sociedade como um todo [Cavalcante 2006], mesmo que não se trate aqui de pesquisa de ponta.

É por isso que, dentre as diversas possibilidades pedagógicas de trabalho com a Wikipédia, pareceu-nos adequado e promissor associá-lo à extensão, que historicamente no Brasil tem sido o mais discreto dos três ramos da atividade universitária, em destaque bem menor do que o ensino e a pesquisa [Jezine 2004]. Desde 2011 têm sido feitas experiências pedagógicas na Unirio com disciplinas de graduação em que alunos editam artigos na Wikipédia [Marques 2012; 2013], mas ainda dentro do contexto tradicional da sala de aula. Em 2012 começaram as atividades do projeto de extensão "Wikipédia na Universidade", em conjunto com a Pró-Reitoria de Extensão e Cultura da Unirio, em que diferentes atividades têm sido desenvolvidas com um público-alvo mais amplo, a partir da experiência docente com a Wikipédia. Entre as iniciativas que a Wikimedia Foundation tem acompanhado ${ }^{5}$, esta é pioneira no trabalho com extensão universitária, e espera-se que sua manutenção e aperfeiçoamento possa servir como estímulo para inciativas semelhantes.

\section{Métodos}

Durante o ano de 2012 foram organizadas as seguintes atividades dentro do projeto de extensão universitária com a Wikipédia na Unirio: a) um curso com carga horária de $60 \mathrm{~h}$; b) uma oficina com carga horária de $4 \mathrm{~h}$, como parte da programação da Semana Nacional de Ciência e Tecnologia, juntamente com a Semana de Integração Acadêmica da universidade; e c) um workshop de $8 \mathrm{~h}$, ministrado fora das dependências da Unirio, para capacitação de professores e "embaixadores". O mesmo modelo usado nos três eventos também seria adaptado para um minicurso em três dias (carga horária de $6 \mathrm{~h}$ ) em 2013, dentro do Encontro Nacional da ANPUH (Associação Nacional de História), para professores de ensino básico e superior. A oficina de 4 h será utilizada aqui como modelo básico para o formato das atividades, já que aumentos na carga horária significam apenas incrementos em detalhes dos pontos trabalhados no workshop mais curto.

A oficina foi realizada em laboratório de informática na biblioteca central da universidade, com um docente, dois embaixadores e 11 estudantes universitários de graduação, a maior parte alunos do curso de História, matriculados em diversos semestres $^{6}$. O recorte específico no público-alvo foi fruto de uma divulgação mais restrita do evento, por ter a oficina se mostrado tecnicamente viável a apenas poucos dias da Semana de Integração Acadêmica. Como o curso de $60 \mathrm{~h}$ e o workshop de $8 \mathrm{~h}$ tiveram inscritos de proveniências diversas, deve-se atentar no futuro para as diferentes formas de aplicação da atividade em diferentes tipos de público-alvo.

A atividade foi estruturada em torno de dois blocos:

\footnotetext{
5 https://outreach.wikimedia.org/wiki/Education_Portal/Projects_and_Programs. Acessado em 30 de setembro de 2013.

6 https://pt.wikipedia.org/wiki/Usuária:Domusaurea/Oficina_de_edição_(4h). Acessado em 30 de setembro de 2013.
} 
1. Apresentação expositiva:

a. O projeto da Wikimedia Foundation e o programa desenvolvido na universidade;

b. O que é a Wikipédia: estrutura de navegação do site e apresentação de alguns de seus recursos principais: abas "discussão", "editar" e "ver histórico"; menu esquerdo: "Esplanada", "Ajuda", "Mudanças recentes", "Páginas novas" e interwikis (páginas correspondentes à visitada nas Wikipédias em outras línguas);

c. Os cinco pilares, com vários exemplos ilustrando as questões levantadas por cada pilar. A escolha dos artigos que ilustraram as questões envolvidas na definição dos pilares foi um dos pontos em que os embaixadores mais participaram, já que conheciam a fundo o histórico e as dinâmicas da Wikipédia lusófona.

2. Atividade prática, derivada da apresentação do quinto pilar, "Seja audaz":

a. Os alunos registram-se no site e aprendem os primeiros passos na edição das páginas wiki através da abertura de suas páginas de usuário (PUs), bem como aprendem a deixar mensagens nas páginas de discussão dos outros usuários (PDUs) - a princípio, para os colegas em sala. Tal atividade foi bastante motivadora, em especial com o uso dos userboxes $^{7}$, com que os editores customizam suas páginas de usuário, definindo gostos pessoais, em uma versão mais livre e simples de páginas de redes sociais;

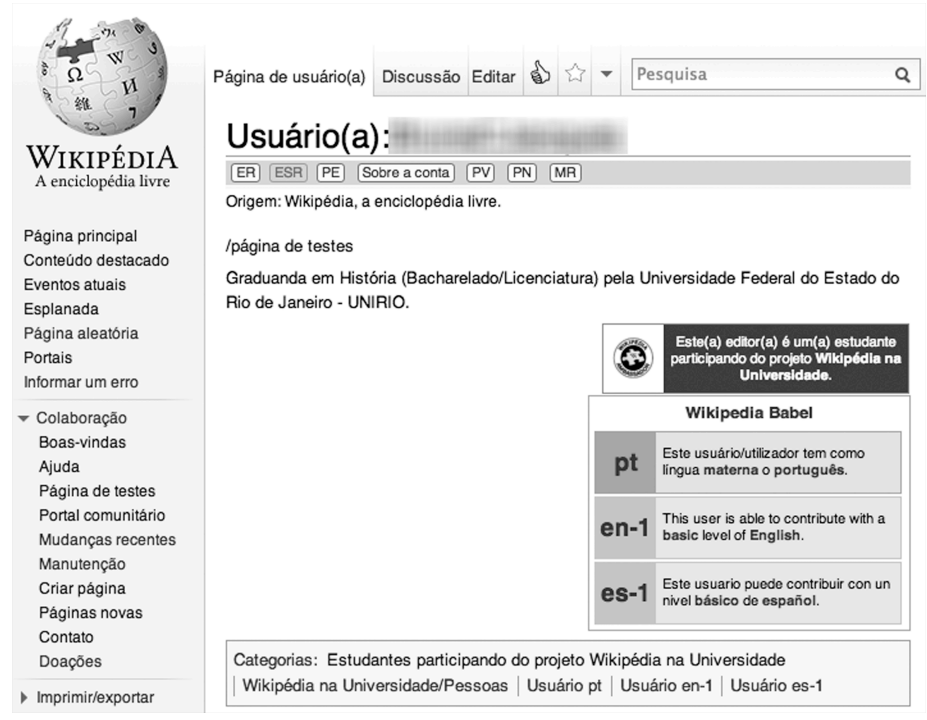

Figura 1: Página de usuário com userboxes criada na oficina

b. Sabendo-se o número prévio dos alunos inscritos, foi preparado de antemão um grupo de artigos para cada dois ou três alunos trabalharem

\footnotetext{
${ }^{7}$ https://pt.wikipedia.org/wiki/Wikipédia:Userbox. Acessado em 30 de setembro de 2013.
} 
conjuntamente, à escolha, que eram artigos simples ("esboços", ou stubs) ainda não disponíveis em português. Os artigos, porém, já deveriam conter originalmente os principais elementos que permitem detectar um padrão aceitável de confiabilidade (referências a materiais como livros, artigos acadêmicos, jornais e sites institucionais). Os textos foram traduzidos e disponibilizados na oficina em arquivos simples de texto (.txt), nos computadores dos alunos. Em seguida, criadas as páginas de teste dentro de suas PUs, onde os alunos poderiam errar sem receios, aos textos são incorporados os códigos wiki das referências, bem como a estrutura padrão do artigo (introdução, sub-tópicos, links internos e externos, bibliografia em ABNT e referências) e imagens do repositório Wikimedia Commons ${ }^{9}$. O trabalho do docente é auxiliado pelos embaixadores, que acompanham os alunos em seus computadores, tirando dúvidas e ajudando a editar.

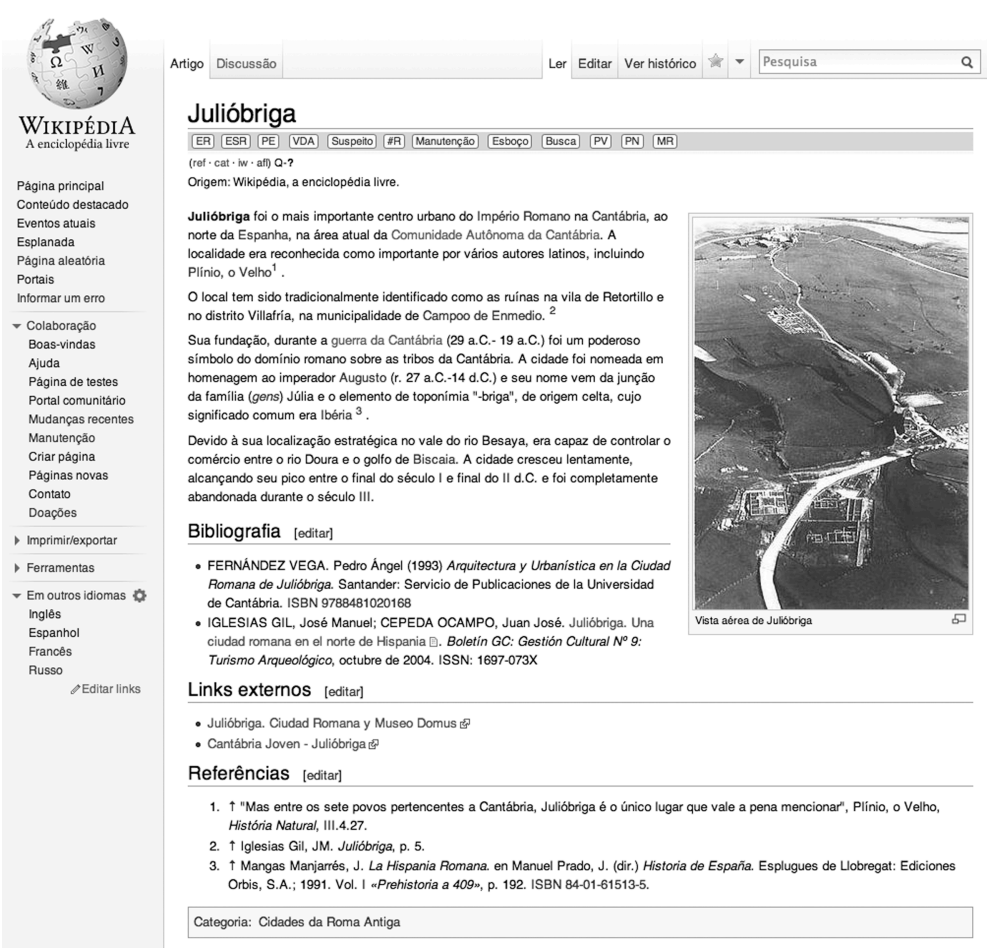

Figura 2: Artigo final da oficina, com imagem, bibliografia, links e referências.

\section{Resultados e discussão}

Foram produzidos na oficina de $4 \mathrm{~h}$ cinco artigos novos na Wikipédia lusófona, todos conferidos e aprovados em tempo real pela comunidade de editores que monitora as novas páginas ${ }^{10}$. Esse monitoramento tem como objetivo evitar vandalismo e a criação

\footnotetext{
${ }^{8}$ https://pt.wikipedia.org/wiki/Wikipédia:Esboço. Acessado em 30 de setembro de 2013.

${ }^{9}$ https://commons.wikimedia.org/wiki/Página_principal. Acessado em 30 de setembro de 2013.

${ }^{10}$ https://pt.wikipedia.org/wiki/Especial:Páginas_novas. Acessado em 30 de setembro de 2013.
} 
de páginas impróprias dentro do conceito do primeiro pilar, como currículos, manuais e blogs, ou textos plagiados, mas é alvo de constantes e acalorados debates entre os editores por causa de diferenças no entendimento da relevância enciclopédica de determinados assuntos [Rosado 2007; Serrano 2011; D'Andréa 2012]. A vantagem de se criar um artigo novo a partir do esboço traduzido é que os alunos não precisam se preocupar com o conteúdo, o que seria uma carga de trabalho inviável para até $8 \mathrm{~h}$ de oficina sem preparação prévia. Todos saíram da oficina muito motivados por ver seu trabalho pronto e no ar, já dominando, portanto, os pré-requisitos para abrir uma nova página corretamente e evitando, assim, discussões com usuários experientes sobre eventuais inadequações de conteúdo e forma. Nos cursos longos, como os de semestres inteiros, é possível ainda trabalhar assuntos mais específicos de interesse de cada aluno, como suas pesquisas de iniciação científica, monografia de fim de curso ou pósgraduação [Marques 2012; 2013].

Uma parte importante da atividade, expansível conforme o tempo do workshop, é o momento em que todos os alunos fazem uma revisão crítica dos trabalhos dos seus colegas ainda nas páginas de teste, o que reproduz em um ambiente seguro e orientado as eventuais críticas que podem ser feitas por editores anônimos externos. Isso é importante porque são diversos os levantamentos e estudos que apontam um baixo nível de retenção de novos editores por causa de tratamentos hostis da comunidade de editores frequentes, que não têm como medir a diferença entre um vândalo eventual e um novato compromissado com um trabalho correto [Serrano 2009; D'Andréa 2012; Britt 2013]. Em cursos mais longos, essa atividade pode ser mais extensa e detalhada, com a leitura crítica e correção de artigos após leituras prévias de textos de suporte e aulas expositivas. Uma parte importante do trabalho nesses cursos mais longos é uma apresentação oral ou escrita do aluno mostrando o estado e os problemas técnicos e de conteúdo de artigos existentes que eles vão trabalhar [Cummings 2009].

Oficinas como essas podem ser montadas para qualquer área do conhecimento, dado que ainda são grandes as deficiências de conteúdo em todas as áreas na Wikipédia lusófona. Porém, acreditamos que a dinâmica funciona melhor se os artigos escolhidos forem próximos da especialidade do/a professor/a ministrante, já que ele/ela pode sugerir no momento da atividade correções e adições ao artigo inicial.

O caráter de extensão das atividades realizadas ainda é incipiente, devido aos primórdios da divulgação do projeto. Pretende-se aperfeiçoar a metodologia utilizada com a participação de estudantes universitários das mais diversas áreas do conhecimento e de outros alunos provenientes de fora do ambiente acadêmico.

\section{Conclusões}

A Wikimedia Foundation iniciou o Wikipedia Education Program ${ }^{11}$ com o intuito de angariar e manter novos editores, procurando as universidades como locus privilegiado de trabalho com qualidade e rigor. No entanto, percebe-se que o nível de retenção de editores nessas atividades pedagógicas com a Wikipédia não é tão diferente da proporção entre usuários e editores frequentes [Roth et al. 2013] - estes na versão lusófona não passam de $5 \%$ dos usuários. De fato, essa desproporção está na própria

\footnotetext{
${ }^{11} \mathrm{http} / / /$ outreach.wikimedia.org/wiki/Wikipedia_Education_Program. Acessado em 30 de setembro de 2013.
} 
raiz do projeto, na medida em que o trabalho é essencialmente voluntário. Nem todos os usuários têm tempo ou disposição para se dedicar a editar artigos, o que se revela como um trabalho minucioso, difícil em termos da adequação da escrita a exigências de clareza e objetividade, além do levantamento de referências. Somado a isso, o mundo acadêmico tem especificidades que são bem distantes do modelo de trabalho da Wikipédia, pois se valoriza a atividade autoral, opinativa e original. No entanto, as competências e habilidades exercitadas na atividade com a Wikipédia são úteis também como base sólida para tais requisitos.

Em última instância, uma oficina de 4 h não irá necessariamente criar um editor da Wikipédia, mas tornará o usuário mais capacitado e crítico, e o fará um multiplicador na medida em que ele saberá explicar a outros usuários como ela funciona. Quanto ao papel da extensão universitária como um trabalho para a sociedade [Cavalcante 2006], é mister lembrar, sem o risco do exagero, que o resultado da atividade reverte essencialmente para toda a população lusófona que acessa a internet no mundo.

\section{Referências}

Ayers, P., Matthews, C., \& Yates, B. (2008). How Wikipedia works: And how you can be a part of it. No Starch Press.

Barbosa, Leonardo P. F.; Oeiras, Janne Yukiko Yoshikawa. (2008) Uso de Wikis em Projetos Escolares: experiências colaborativas com alunos de ensino fundamental. In: Anais do XXVIII Congresso da SBC/WIE-Workshop de Informática na Escola. Belém-PA.

Bottentuit Jr., João Batista; Coutinho, Clara Pereira. (2008) Wikis em educação: potencialidades e contextos de utilização. Actas do Encontro sobre Web 2.0, Braga. Disponível em http://hdl.handle.net/1822/8460.

Britt, Lori L. (2013) Writing for Wikipedia: co-constructing knowledge and writing for a public audience. In: FERRIS, Sharmila; WILDER, Hilary (eds.) The Plugged-in Professor: Tips and techniques for teaching with social media. Cambridge: Chandos Publishing.

Campos, Aline de. (2009) Conflitos na colaboração: um estudo das tensões em processos de escrita coletiva na web 2.0. Dissertação de Mestrado - UFRGS. Disponível em http://hdl.handle.net/10183/16928.

Casebourne et al. (2012) Assessing the accuracy and quality of Wikipedia entries compared to popular online encyclopedias. A comparative preliminary study across disciplines in English, Spanish and Arabic. Epic, Brighton, UK. Disponível em http://upload.wikimedia.org/wikipedia/commons/2/29/EPIC_Oxford_report.pdf.

Cavalcante, Lídia Eugênia. (2006) Políticas de formação para a competência informacional: o papel das universidades. Revista Brasileira de Biblioteconomia e Documentação, Nova Série. São Paulo, v.2, n.2, p.47-62. Disponível em http://rbbd.febab.org.br/rbbd/article/view/17/5.

Costa, Rodrigo Garrett da; Passerino, Liliana Maria; Araújo, Thiago Moreira de Resende. (2011) Aprendizagem colaborativa baseada em wiki no estudo de conceitos 
da termodinâmica. In: Anais do XVII WIE-Workshop de Informática na Escola. Aracaju-SE, p. 1178-1187.

Cummings (2009), Robert E. Lazy Virtues: Teaching Writing in the Age of Wikipedia. Nashville: Vanderbilt University Press.

D’Andréa, Carlos F. B.(2012) Edição colaborativa na Wikipédia: desafios e possibilidades. In: Moura, Maria Aparecida. (Org.). Formação de pesquisadores juvenis: abordagens teóricas e metodológicas. Belo Horizonte: PROEX UFMG, p. 235-246.

Dudziak, Elizabeth Adriana. (2008) Os faróis da sociedade de informação: uma análise crítica sobre a situação da competência em informação no Brasil. Informação e Sociedade: Estudos. João Pessoa, v.18, n.2, p. 41-53. Disponível em http://www.producao.sibi.usp.br/handle/2012.1/16882.

Gomes, Maria Rodrigues. (2007) A ferramenta wiki: uma experiência pedagógica. Comunicação \& Educação, XVII, $\mathrm{n}^{\circ}$ 2. Disponível em http://www.revistasusp.sibi.usp.br/pdf/ced/v12n2/v12n2a12.pdf.

Jesus, Ana Maria Ribas de. (2011) Wiki: ferramenta de autoria e colaboração na Web 2.0. In: Anais do IV Encontro Nacional de Hipertexto e Tecnologias Educacionais. $\quad$ UNISO, 2011. http://www.uniso.br/ead/hipertexto/anais/07_AnaRibas1.pdf.

Jezine, E. (2004). As práticas curriculares e a extensão universitária. In: Anais do II Congresso Brasileiro de Extensão Universitária. Belo Horizonte, pp. 21-32.

Lampe, C., Obar, J., Ozkaya, E., Zube, P., \& Velasquez, A. (2012, February). Classroom Wikipedia participation effects on future intentions to contribute. In: Proceedings of the ACM 2012 conference on Computer Supported Cooperative Work, pp. 403-406. ACM.

Marques, Juliana Bastos. (2012) A História Romana na Wikipédia. In: E-book da 2a. Conferência Online de Informática Educacional. Porto: Universidade Católica Editora, p. 237-240.

(2013) Trabalhando com a história romana na Wikipédia: uma experiência em conhecimento colaborativo na universidade. Revista História Hoje, no prelo.

Obar, Jonathan A.; Roth, Amy. (2011) The Wikipedia Public Policy Initiative: Exploring the Potential Benefits of Using Wikipedia in the University Classroom as a Tool for Innovative E-Pedagogy. Social Science Research Network. Disponível em http://ssrn.com/abstract=1923888.

Rosado, Luiz Alexandre da Silva. (2007) Autoria textual coletiva fora do âmbito acadêmico: delineando o perfil dos wikipedistas. $1^{\circ}$ Colóquio de Pesquisas em Educação e Mídia: diálogo entre culturas, PUC-Rio/UNIRIO, Rio de Janeiro - RJ. Disponível em http://alexandrerosado.net/attachments/005_Coloquio1AlexandreRosado.pdf.

Roth, Amy; Davis, Rochelle; Carver, Brian. (2013) Assigning Wikipedia editing: Triangulation toward understanding university student engagement. First Monday, v. 18, n. 6, 2013. 
Salsano, Alfredo. (2000) Enciclopédia. In: Romano, Ruggiero (coord.) Enciclopédia Einaudi. Vol. 41: Conhecimento. Lisboa: Imprensa Nacional-Casa da Moeda, 2000, p. 369-432.

Santos, J. D.; Pandini, M. J. (2009) Wikipédia: descrição dos métodos de organização e recuperação da Enciclopédia Colaborativa. In: Anais do Congresso Brasileiro de Biblioteconomia, Documentação e Ciência da Informação. Bonito: CBBD XXIII.

Saorín Pérez, Tomás; de Haro y de San Mateo, María Verónica; Pastor Sánchez, Juan Antonio. (2011) Posibilidades de Wikipedia en la docencia universitaria: elaboración colaborativa de conocimiento. Ibersid - Revista de sistemas de información y documentación, vol. 5, p. 89-97. Disponível em http://ibersid.eu/ojs/index.php/ibersid/article/view/3915.

Serrano, Paulo H. (2009) Participações em uma comunidade de prática; O sustentáculo da Wikipédia. In: III Encontro Nacional sobre Hipertexto. Belo Horizonte. Anais Hipertexto. Disponível em http://www.ufpe.br/nehte/hipertexto2009/anais/pw/participacoes-em-uma-comunidade.pdf.

Serrano, Paulo Henrique. (2011) Coerência entre princípios e práticas na Wikipédia lusófona: Uma análise semiótica. Dissertação de Mestrado - UFMG, 2011. Disponível em http://dspace.lcc.ufmg.br/dspace/handle/1843/DAJR-8M6R2F. Acessos em 28 de agosto de 2012.

Silva, Aletéia Karina Lopes da; Domingues, Maria José Carvalho de Souza. (2008) Plágio no meio acadêmico: de que forma alunos de pós-graduação compreendem o tema. Perspectivas Contemporâneas, Campo Mourão, v. 3, n. 2, p. 117-135. Disponível

em http://www.revista.grupointegrado.br/revista/index.php/perspectivascontemporaneas/ article/viewFile/448/247.

Souza, Cristina Gomes de. (2007) Plágio em trabalhos acadêmicos: uma pesquisa com alunos de graduação. In: Anais do XXVII Encontro Nacional de Engenharia de Produção, ABEPRO, Foz do Iguaçu. Disponível em http://www.abepro.org.br/biblioteca/ENEGEP2007_TR660482_9513.pdf.

Soylu, Firat. (2009) Academics' Views On and Uses of Wikipedia. GNOVIS, vol. IX, n. 2, Spring. Disponível em http://gnovisjournal.org/2009/05/13/academics-viewsand-uses-wikipedia. Acesso em 28 de agosto de 2012.

Vieira, Marli Fátima Vick. (2008) A Wikipédia é confiável? Credibilidade, utilização de uma enciclopédia online no ambiente escolar. Dissertação (Mestrado em Educação) - Univale - Santa Catarina, 2008. Disponível em http://siaibib01.univali.br/pdf/Marli\%20Fatima\%20Vick\%20Vieira.pdf.

Watts, Linda S. (2012). Writing Radical Lives: Undergraduates Publishing Activist Biographies on Wikipedia. Cutting-edge Technologies in Higher Education, Vol. Iss: 6 Part A, pp.109 - 135 . 